\title{
A new analysis of the MEGA M 31 microlensing events
}

\author{
G. Ingrosso ${ }^{1}$, S. Calchi Novati ${ }^{2}$, F. De Paolis ${ }^{1}$, Ph. Jetzer ${ }^{3}$, A. A. Nucita ${ }^{1}$, G. Scarpetta ${ }^{2}$, and F. Strafella ${ }^{1}$ \\ 1 Dipartimento di Fisica, Università di Lecce and INFN, Sezione di Lecce, CP 193, 73100 Lecce, Italy \\ e-mail: nucita@le.infn.it \\ 2 Dipartimento di Fisica "E. R. Caianiello", Università di Salerno, 84081 Baronissi (SA) and INFN, Sezione di Napoli, Italy \\ ${ }^{3}$ Institute for Theoretical Physics, University of Zürich, Winterthurerstrasse 190, 8057 Zürich, Switzerland
}

Received 16 June 2006 / Accepted 3 October 2006

\section{ABSTRACT}

\begin{abstract}
We discuss the results of the MEGA microlensing campaign towards M31. Our analysis is based on analytically evaluating the microlensing rate, taking the observational efficiency into account as given by the MEGA collaboration. In particular, we studied the spatial and time-duration distributions of the microlensing events for several mass distribution models of the M 31 bulge. We find that only for extreme models of the M31 luminous components is it possible to reconcile the total observed MEGA events with the expected self-lensing contribution. Nevertheless, the expected spatial distribution of self-lensing events is more concentrated and hardly agrees with the observed distribution. We thus find it difficult to explain all events as due to self-lensing alone. On the other hand, the small number of events does not yet allow firm conclusions to be drawn on the halo dark matter fraction in the form of MACHOs.
\end{abstract}

Key words. gravitational lensing - Galaxy: halo - galaxies: individuals: M31

\section{Introduction}

Since the proposal by Paczyński (1986), gravitational microlensing has proved to be an efficient tool for studying the MACHO contribution to the dark matter galactic halos. The first line of sight to be explored with this purpose has been that towards the Magellanic Clouds (Alcock et al. 1993; Aubourg et al. 1993; Udalski et al. 1993). As first discussed by Crotts (1992), Baillon et al. (1993), and Jetzer (1994), observations towards M 31 have also been undertaken (Crotts \& Tomaney 1996; Ansari et al. 1997).

The interpretation of the results obtained so far remains, however, debated and controversial. Along the line of sight towards the LMC the MACHO collaboration, Alcock et al. (2000) reported the signal of a halo fraction of about $20 \%$ in the form of MACHOs with mass $\simeq 0.5 M_{\odot}$, while the latest results of the EROS collaboration towards both the SMC and the LMC (Afonso et al. 2003; Tisserand 2005; Tisserand et al. 2006) are even compatible with a no MACHO hypothesis.

The case towards M 31 is complicated by further degeneration in the lensing parameter space due to the fact that sources at baseline are unresolved, a situation referred to as "pixel-lensing" (Crotts 1992; Baillon et al. 1993; Gould 1996). Still, a handful of microlensing events have been observed in the meantime (Ansari et al. 1999; Auriere et al. 2001; Calchi Novati et al. 2002, 2003; Paulin-Henriksson et al. 2003; Riffeser et al. 2003; de Jong et al. 2004; Belokurov et al. 2005; Joshi et al. 2005), and lately the first constraints on the halo fraction have been reported. The results of the POINT-AGAPE collaboration (Calchi Novati et al. 2005) are compatible with the ones of the MACHO group, by putting a lower limit on the halo fraction in the form of MACHOs of $\sim 20 \%$ for objects in the mass range $0.5-1 M_{\odot}$. In contrast, the MEGA collaboration (de Jong et al. 2006) finds that their results, although not conclusive, agree with a no MACHO hypothesis. Although the issues involved in the microlensing observations towards the LMC or the M31 are indeed rather different, the results for the halo fraction in the form of MACHOs depend crucially on the prediction of the expected signal due to known luminous populations, this being dominated by the "selflensing" signal where both source and lens belong to same star population residing respectively either in the LMC or in M 31 . This problem is indeed the main aspect we want to discuss in this paper.

The issue of the expected microlensing signal towards M 31 has been discussed in a few works (e.g. Kerins et al. 2001; Baltz et al. 2003; Riffeser et al. 2006; Kerins et al. 2006). In this respect the modeling of the M31 luminous components is an essential aspect to be dealt with in order to get meaningful results. We first considered these aspects in De Paolis et al. (2005); then more recently in Ingrosso et al. (2006), we developed a Monte Carlo simulation that we used to investigate the nature and location of the microlensing candidate events towards M 31 as reported in a first paper by the MEGA collaboration (de Jong et al. 2004). In the present work our aim is to explore these issues further taking the latest MEGA results into account (de Jong et al. 2006). In particular, we now go through a full characterisation of the expected signal, including the predicted number of events, which we then compare with the observational results. Our aim is to explore the question of whether the expected selflensing signal due to stars belonging either to the bulge or the disc of M 31 is able, as claimed by de Jong et al. (2006), to fully explain their results.

The plan of the paper is as follows. In Sect. 2 we describe the microlensing rate, our main tool of investigation, and present its predictions. In Sect. 3 we critically discuss the models used to describe the different galactic components involved. In Sect. 4 we discuss our main results and give some concluding remarks.

\section{Event rate prediction}

In evaluating ${ }^{1}$ the expected event number along a fixed line of sight, we take the existence of two source populations into

1 Here we follow the derivation in our previous paper Ingrosso et al. (2006) with some modifications. 
Table 1. Position, magnitude at maximum $\Delta r$, and full-width halfmaximum duration $t_{1 / 2}$ for the 14 MEGA events.

\begin{tabular}{ccccr}
\hline \hline MEGA & $\begin{array}{c}X^{a} \\
\text { arcmin }\end{array}$ & $\begin{array}{c}Y \\
\text { arcmin }\end{array}$ & \multicolumn{1}{c}{$\begin{array}{c}\Delta r \\
\mathrm{mag}\end{array}$} & \multicolumn{1}{c}{\begin{tabular}{c}
\multicolumn{1}{c}{ day } \\
day
\end{tabular}} \\
\hline 1 & -4.367 & -2.814 & $21.8 \pm 0.4$ & $5.4 \pm 0.7$ \\
2 & -4.478 & -3.065 & $21.51 \pm 0.06$ & $4.2 \pm 0.7$ \\
3 & -7.379 & -1.659 & $21.6 \pm 0.1$ & $2.3 \pm 2.9$ \\
7 (N2) & -21.164 & -6.248 & $19.37 \pm 0.02$ & $17.8 \pm 0.4$ \\
8 & -21.650 & +7.670 & $22.3 \pm 0.2$ & $27.5 \pm 1.2$ \\
9 & -33.833 & -2.251 & $21.97 \pm 0.08$ & $2.3 \pm 0.4$ \\
10 & -3.932 & -13.847 & $22.2 \pm 0.1$ & $44.7 \pm 5.6$ \\
$11(\mathrm{~S} 4)$ & +19.193 & -11.833 & $20.72 \pm 0.03$ & $2.3 \pm 0.3$ \\
13 & +22.072 & -22.022 & $23.3 \pm 0.1$ & $26.8 \pm 1.5$ \\
14 & +19.349 & -29.560 & $22.5 \pm 0.1$ & $25.4 \pm 0.4$ \\
15 & -6.634 & -0.697 & $21.63 \pm 0.08$ & $16.1 \pm 1.1$ \\
16 (N1) & -6.886 & +3.843 & $21.16 \pm 0.06$ & $1.4 \pm 0.1$ \\
17 & +21.214 & -5.161 & $22.2 \pm 0.1$ & $10.1 \pm 2.6$ \\
18 & +6.995 & -13.533 & $22.7 \pm 0.1$ & $33.4 \pm 2.3$ \\
\hline
\end{tabular}

${ }^{a}$ The coordinate system we adopt has its origin in the M31 centre and the $X$ axis oriented along the M 31 disk major axis.

account (stars in the M31 bulge and disk) with number density $n_{\mathrm{s}}\left(D_{\mathrm{os}}, M\right)$ and of five lens populations (stars in the M 31 bulge, stars in the M31 and MW disks, MACHOs in M31 and MW halos) with density $n_{\mathrm{l}}\left(D_{\mathrm{ol}}, \mu\right)$. Here $D_{\mathrm{os}}\left(D_{\mathrm{ol}}\right)$ is the source (lens) distance from the observer, $M$ the source magnitude, and $\mu$ the lens mass in solar units.

We assume, as usual, that the mass distribution of the lenses is independent of their position in M31 or in the Galaxy (factorization hypothesis), so the lens number density (per unit of volume and mass) $n_{1}\left(D_{\mathrm{ol}}, \mu\right)$ can be written as (Jetzer et al. 2002)

$n_{1}\left(D_{\mathrm{ol}}, \mu\right)=\left(\frac{\rho_{\mathrm{l}}\left(D_{\mathrm{ol}}\right)}{\rho_{\mathrm{l}}(0)}\right) \psi_{0}(\mu)$,

where $\rho_{1}(0)$ is the local mass density of the considered lens population in the Galaxy or the central density in $\mathrm{M} 31, \psi_{0}(\mu)$ the corresponding lens number density per unit mass. The normalisation is given by

$$
\int_{\mu_{\min }}^{\mu_{\text {up }}} \psi_{0}(\mu) \mu \mathrm{d} \mu=\frac{\rho_{\mathrm{l}}(0)}{M_{\odot}} .
$$

Here $\mu_{\min }$ and $\mu_{\mathrm{up}}$ are the lower and the upper limits for the lens masses (see Sect. 3.2).

Likewise, assuming that the magnitude distribution of the sources is independent of their position in M31, the source number density (per unit of volume and magnitude) $n_{\mathrm{s}}\left(D_{\mathrm{os}}, M\right)$ can be written as

$n_{\mathrm{s}}\left(D_{\mathrm{os}}, M\right)=\left(\frac{\mathcal{L}_{\mathrm{s}}\left(D_{\mathrm{os}}\right)}{\mathcal{L}_{\mathrm{s}}(0)}\right) \phi_{\mathrm{s}}(M)$

where $\mathcal{L}_{\mathrm{s}}(0)$ is central luminosity density of the considered source population, $\phi_{\mathrm{s}}(M)$ the source number density per unit magnitude in the M 31 centre, and the normalisation now reads

$\int_{M_{\min }}^{M_{\text {up }}} \phi_{\mathrm{s}}(M) L(M) \mathrm{d} M=\mathcal{L}_{\mathrm{s}}(0)$.

Here $M_{\min }$ and $M_{\text {up }}$ are the lower and the upper limits for the source magnitude (see Sect. 3.1), $L(M)$ is the luminosity in a given photometric band

$L(M)=\eta_{\text {Vega }} L_{\odot} 10^{-M / 2.5}$, $\eta_{\text {Vega }}$ being the Vega luminosity (in solar units) in the considered band.

We consider the volume element of the microlensing tube to be $\mathrm{d}^{3} x=\left(v_{\mathrm{r} \perp} \cdot \boldsymbol{n}\right) R_{\mathrm{E}} u_{\mathrm{th}} \mathrm{d} \alpha \mathrm{d} D_{\mathrm{ol}}$, where $R_{\mathrm{E}}$ is the Einstein radius, $v_{\mathrm{r} \perp}$ the relative tranverse velocity between the lens and the microlensing tube, with distribution function $f\left(\boldsymbol{v}_{\mathrm{r} \perp}\right), \boldsymbol{n}$ the inner normal to the microlensing tube and $\alpha$ the angle between $\boldsymbol{n}$ and $\boldsymbol{A}_{\perp}$ (see Eq. (8)). Assuming perfect observational sensitivity to microlensing, the differential event rate $\mathrm{d} N_{\mathrm{ev}} / \mathrm{d} \Omega$ (in units of event $\mathrm{sr}^{-1}$ ) for microlensing by compact objects with an impact parameter below a certain threshold $u_{\text {th }}$, during the time interval $\mathrm{d} t$, is given by (Griest 1991; De Rújula et al. 1991)

$$
\begin{aligned}
\frac{\mathrm{d} N_{\mathrm{ev}}}{\mathrm{d} \Omega}= & D_{\mathrm{os}}^{2} u_{\mathrm{th}} R_{\mathrm{E}} \mathrm{d} \alpha v_{\mathrm{r} \perp} f\left(\boldsymbol{v}_{\mathrm{r} \perp}\right) \mathrm{d}^{2} \boldsymbol{v}_{\mathrm{r} \perp} \cos \theta \\
& n_{\mathrm{l}}\left(D_{\mathrm{ol}}, \mu\right) n_{\mathrm{s}}\left(D_{\mathrm{os}}, M\right) \mathrm{d} \mu \mathrm{d} M \mathrm{~d} D_{\mathrm{os}} \mathrm{d} D_{\mathrm{ol}} \mathrm{d} t,
\end{aligned}
$$

where $\theta \in(-\pi / 2, \pi / 2)$ is the angle between $\boldsymbol{n}$ and $\boldsymbol{v}_{\mathrm{r} \perp}$. We assume that the velocity distributions of lenses and sources are isotropic around their streaming velocities (if present) due to the rotation of the considered population with respect to the M 31 or MW centre (we neglect any transverse drift velocity of the M31 centre with respect to the Galaxy). Accordingly, the lens (source) velocity is split into a random component - which follows a Maxwellian distribution with one-dimensional velocity dispersion $\sigma_{1}\left(\sigma_{\mathrm{s}}\right)$ - and a streaming component, namely $\boldsymbol{v}_{\mathrm{l}}=\boldsymbol{v}_{\mathrm{l} \text {,ran }}+\boldsymbol{v}_{\mathrm{l} \text {,rot }}$ and $\boldsymbol{v}_{\mathrm{s}}=\boldsymbol{v}_{\mathrm{s} \text {,ran }}+\boldsymbol{v}_{\mathrm{s} \text {, rot }}$. When the lens and source velocities are projected onto the lens plane (transverse to the microlensing tube), the respective random velocity distributions are again described by Maxwellian functions, with the same one-dimensional velocity dispersion $\sigma_{1}$ for lenses, and with (projected) dispersion $\left(D_{1} / D_{\mathrm{s}}\right) \sigma_{\mathrm{s}}$ for sources. Then, neglecting the streaming, the relative, projected, random velocity $\boldsymbol{v}_{\mathrm{ls} \perp \text {, ran }}=\boldsymbol{v}_{1 \perp \text {,ran }}-\boldsymbol{v}_{\mathrm{s} \perp \text {,ran }}$ of lenses and sources is a Maxwellian distribution $f\left(\boldsymbol{v}_{\mathrm{ls} \perp, \mathrm{ran}}\right)$ with combined width

$\sigma_{\mathrm{s} 1}=\sqrt{\sigma_{1}^{2}+\left(D_{\mathrm{ol}} / D_{\mathrm{os}}\right)^{2} \sigma_{\mathrm{s}}^{2}}$

We now include all streaming motions in the vector $\boldsymbol{A}_{\perp}$ defined as the difference between the projected, streaming velocities of lenses, sources, and observer, namely

$\boldsymbol{A}_{\perp}=\left(1-\frac{D_{\mathrm{ol}}}{D_{\mathrm{os}}}\right) \boldsymbol{v}_{\odot \perp, \mathrm{rot}}+\left(\frac{D_{\mathrm{ol}}}{D_{\mathrm{os}}}\right) \boldsymbol{v}_{\mathrm{s} \perp \text {, rot }}-\boldsymbol{v}_{\mathrm{l} \perp \text {, rot }}$.

The resulting distribution function $f\left(v_{\mathrm{r} \perp}\right)$ of the relative, transverse velocity between the lenses and the microlensing tube is now given by the Maxwellian function $f\left(\boldsymbol{v}_{\mathrm{ls} \perp \text {,ran }}\right)$ shifted by the vector $\boldsymbol{A}_{\perp}$, which we write in polar coordinates on the lens plane as

$f\left(\boldsymbol{v}_{\mathrm{r} \perp}\right) \mathrm{d}^{2} \boldsymbol{v}_{\mathrm{r} \perp}=\frac{1}{2 \pi \sigma_{\mathrm{sl}}^{2}} \mathrm{e}^{-\frac{\left(\boldsymbol{v}_{\mathrm{r} \perp}-\boldsymbol{A}_{\perp}\right)^{2}}{2 \sigma_{\mathrm{sl}}^{2}}} v_{\mathrm{r} \perp} \mathrm{d} v_{\mathrm{r} \perp} \mathrm{d} \theta$.

Taking $\alpha$ to be the angle between $\boldsymbol{A}_{\perp}$ and the normal $\boldsymbol{n}$ to the microlensing tube, it turns out that $\varphi=\alpha+\theta$, where $\varphi$ is the angle between $\boldsymbol{v}_{\mathrm{r} \perp}$ and $\boldsymbol{A}_{\perp}$.

We recall that in the pixel-lensing regime the effective radius of the microlensing tube is a function of the source star magnitude, namely $u_{\mathrm{th}}=u_{\mathrm{th}}(M)$. Moreover in the following, we evaluate the differential rate taking an efficiency function into account that depends on the impact parameter, $\epsilon=\epsilon\left(u_{\mathrm{th}}\right)$. Therefore, we are going to replace $\mathrm{d} N_{\mathrm{ev}}$ by $\int \mathrm{d} N_{\mathrm{ev}} / \mathrm{d} u_{\mathrm{th}} \times \epsilon\left(u_{\mathrm{th}}\right) \mathrm{d} u_{\mathrm{th}}$ in Eq. (6) with upper limit $u_{\mathrm{T}}(M)$. 
Eventually, after integration on the angular variables $\theta$ and $\alpha$, one obtains the expected event number rate (events $\mathrm{sr}^{-1}$ ) during observation time $T_{\mathrm{obs}}$

$$
\begin{aligned}
& \frac{\mathrm{d} N_{\mathrm{ev}}}{\mathrm{d} \Omega}=T_{\mathrm{obs}} 4 \sqrt{2} \sigma_{\mathrm{sl}} \sqrt{\frac{4 G M_{\odot}}{c^{2}}} \int_{0}^{u_{\mathrm{T}}(M)} \mathrm{d} u_{\mathrm{th}} \\
& \quad \times \int_{M_{\min }}^{M_{\mathrm{up}}} \phi_{\mathrm{s}}(M) \mathrm{d} M \int_{\mu_{\mathrm{min}}}^{\mu_{\mathrm{up}}} \mathrm{d} \mu \mu^{1 / 2} \psi_{0}(\mu) \int_{0}^{\infty} D_{\mathrm{os}}^{2} \mathrm{~d} D_{\mathrm{os}} \\
& \quad \times \int_{0}^{D_{\mathrm{os}}} \mathrm{d} D_{\mathrm{ol}} \sqrt{\frac{D_{\mathrm{ol}}\left(D_{\mathrm{os}}-D_{\mathrm{ol}}\right)}{D_{\mathrm{os}}}}\left(\frac{\rho_{\mathrm{l}}\left(D_{\mathrm{ol}}\right)}{\rho_{\mathrm{l}}(0)}\right)\left(\frac{\mathcal{L}_{\mathrm{s}}\left(D_{\mathrm{os}}\right)}{\mathcal{L}_{\mathrm{s}}(0)}\right) \\
& \quad \times \int_{0}^{\infty} z^{2} \mathrm{e}^{-\left(z^{2}+\beta^{2}\right)} I_{0}(2 \beta z) \epsilon\left(t_{1 / 2}, \Delta f\right) \mathrm{d} z
\end{aligned}
$$

where $z=v_{\mathrm{r} \perp} /\left(\sqrt{2} \sigma_{\mathrm{sl}}\right), \beta=\left|\boldsymbol{A}_{\perp}\right| /\left(\sqrt{2} \sigma_{\mathrm{sl}}\right)$, and $I_{0}(2 \beta z)$ is the zero-order modified Bessel function ${ }^{2}$.

In the previous equation we explicitly take an experimental event detection efficiency $\epsilon\left(t_{1 / 2}, \Delta f\right)$ into account, given as a function of the full-width half-maximum event duration $t_{1 / 2}$

$$
\begin{aligned}
& t_{1 / 2}=t_{\mathrm{E}} f(a), \quad a=A_{\max }-1 \\
& f(a)=2 \sqrt{2}\left(\frac{a+2}{\sqrt{a^{2}+4 a}}-\frac{a+1}{\sqrt{a^{2}+2 a}}\right)^{1 / 2},
\end{aligned}
$$

and of the maximum flux difference during a microlensing event

$\Delta f=f_{0}\left(A_{\max }-1\right)$.

Here $t_{\mathrm{E}}$ is the Einstein time, $A_{\max }=A_{\max }\left(u_{\mathrm{th}}\right)$ the amplification at maximum, and $f_{0}$ the unlensed source flux.

It is well known that self-lensing and dark-lensing events may have different time durations, depending on the MACHO mass value. On the other hand, experimental results in pixellensing observations are usually given in terms of the $t_{1 / 2}$ time scale. Thus, it is important to evaluate the expected event rate as a function of $t_{1 / 2}$.

From Eq. (11) and the relation $t_{\mathrm{E}}=R_{\mathrm{E}} / v_{1 \perp}$, it follows that

$t_{1 / 2}=\frac{R_{\mathrm{E}} f(a)}{z \sqrt{2} \sigma_{\mathrm{sl}}}$,

and it is straightforward to derive the differential event rate

$$
\begin{aligned}
& \frac{\mathrm{d}^{2} N_{\mathrm{ev}}}{\mathrm{d} \Omega \mathrm{d} t_{1 / 2}}\left(t_{1 / 2}\right)=T_{\mathrm{obs}} 8 \sigma_{\mathrm{sl}}^{2} \int_{0}^{u_{\mathrm{T}}(M)} \mathrm{d} u_{\mathrm{th}} \\
& \quad \times \int_{M_{\mathrm{min}}}^{M_{\mathrm{up}}} \phi_{\mathrm{s}}(M) \mathrm{d} M \int_{\mu_{\min }}^{\mu_{\mathrm{up}}} \mathrm{d} \mu \psi_{0}(\mu) \int_{0}^{\infty} D_{\mathrm{os}}^{2} \mathrm{~d} D_{\mathrm{os}} \\
& \quad \times \int_{0}^{D_{\mathrm{os}}} \mathrm{d} D_{\mathrm{ol}}\left(\frac{\rho_{\mathrm{l}}\left(D_{\mathrm{ol}}\right)}{\rho_{\mathrm{l}}(0)}\right)\left(\frac{\mathcal{L}_{\mathrm{s}}\left(D_{\mathrm{os}}\right)}{\mathcal{L}_{\mathrm{s}}(0)}\right) \\
& \quad \times z^{4} \mathrm{e}^{-\left(z^{2}+\beta^{2}\right)} I_{0}(2 \beta z) \frac{1}{f(a)} \epsilon\left(t_{1 / 2}, \Delta f\right),
\end{aligned}
$$

where $z$ is now given in terms of $t_{1 / 2}$ and $A_{\max }$ through Eq. (13).

${ }^{2}$ By comparing Eq. (10) with Eqs. (11) and (12) in Ingrosso et al. (2006), one can see that the composition of the two Maxwellian (projected) velocity distributions for lenses and sources permits now to evaluate analytically the two-dimensional integration on the source velocity in Eq. (12).
The model parameters that need to be specified are the luminosity $\phi_{\mathrm{s}}(M)$ and mass $\psi_{0}(\mu)$ functions, the stellar mass distributions in M 31 and MW, the mass-to-luminosity ratios for the stellar populations in $\mathrm{M} 31$, the velocity dispersion $\sigma_{\mathrm{s}}$, and $\sigma_{1}$ for the source and lens populations. More model parameters derive from the consideration of the existence of dark matter in both M 31 and MW halos.

\section{Models}

In this section we briefly describe the source luminosity function for the M31 galaxy together with the lens mass function. We also describe the mass distributions we use for both M31 and Milky Way galaxies.

\subsection{Source luminosity function}

In pixel-lensing experiments, only bright and sufficiently magnified sources can give rise to detectable microlensing events. Monte Carlo simulations (e.g. Ingrosso et al. 2006) allow us to determine the useful range of source magnitude $M_{\min } \simeq-6$ and $M_{\max } \simeq 3$, and the threshold value for the impact parameter $u_{\mathrm{T}}(M)$.

With the lack of precise information about the source luminosity function $\phi_{\mathrm{s}}(M)$ in M 31, we adopt the luminosity function derived for local stars in the Galaxy and assume that it also holds for M 31, irrespective of its position. In particular, following Mamon \& Soneira (1982), the stellar luminosity function in the magnitude range $-6 \leq M \leq 16$ is given by

$\phi_{\mathrm{S}}(M)=H \frac{10^{\beta\left(M-M^{*}\right)}}{\left[1+10^{-(\alpha-\beta) \delta\left(M-M^{*}\right)}\right]^{1 / \delta}}$,

where, in the $R$-band (the observational band of the MEGA collaboration) $M^{*}=1.4, \alpha \simeq 0.74, \beta=0.045$, and $\delta=1 / 3$. The constant $H$ in Eq. (15) is determined via the normalisation condition in Eq. (4), namely

$$
\int_{-6}^{16} \phi_{\mathrm{s}}(M) L(M) \mathrm{d} M=\rho_{\mathrm{s}}(0)\left(\frac{M}{L_{R}}\right)^{-1},
$$

where $\left(M / L_{R}\right)$ is the mass-to-luminosity ratio for the source star population in the $R$-band. Note that the normalisation for the source density distribution, Eq. (20), implies that the event rate does not depend on $\left(M / L_{R}\right)$.

\subsection{Lens mass function}

For lenses belonging to the bulge and disk star populations, the lens mass is assumed to follow a broken power law (Gould et al. 1997)

$$
\begin{aligned}
\psi_{0}(\mu) & =K_{1} \mu^{-0.56} \text { for } \mu_{\min } \leq \mu \leq 0.59 \\
& =K_{2} \mu^{-2.20} \text { for } 0.59 \leq \mu \leq \mu_{\text {up }}
\end{aligned}
$$

where the lower limit $\mu_{\min }=0.1$ and the upper limit $\mu_{\text {up }}$ is 1 for M 31 bulge stars and 1.7 for M 31 and MW disk stars. The constants $K_{1}$ and $K_{2}$ are fixed according to the normalisation condition given by Eq. (2). The resulting mean mass for lenses in the bulges and disks are $\left\langle m_{\mathrm{b}}\right\rangle \sim 0.41 M_{\odot}$ and $\left\langle m_{\mathrm{d}}\right\rangle \sim 0.51 M_{\odot}$, respectively.

We also consider steeper mass function, as proposed by Zoccali et al. (2000), and find that our estimate of the selflensing event number turns out to be rather insensitive to the 
Table 2. The M31 disk and bulge models, with relevant parameters for WeCapp (Riffeser et al. 2006) and our reference model.

\begin{tabular}{c|cccccc|ccccccc}
\hline \hline & \multicolumn{9}{c}{ Bulge } & \multicolumn{1}{c}{ Disk } \\
Model $^{a}$ & $\rho(0)$ & $a_{0}$ & $M$ & $\operatorname{ext}_{R}$ & $\left(M / L_{R}\right)$ & $\sigma$ & $\rho(0)$ & $h$ & $H$ & $M$ & $\operatorname{ext}_{R}$ & $\left(M / L_{R}\right)$ & $\sigma(2 h)$ \\
\hline WeCapp & $3.97 \times 10^{4}$ & & 4.00 & 0.36 & 2.96 & 140 & 0.20 & 6.4 & 0.3 & 3.09 & 0.68 & 0.88 & 40 \\
reference & $4.53 \times 10^{4}$ & $2.62 \times 10^{-3}$ & 3.85 & $-{ }^{b}$ & - & - & - & - & - & - & - & - & - \\
MEGA A $^{c}$ & & & 4.4 & & 3.6 & & & & 1 & 5.5 & & 2.4 \\
\hline
\end{tabular}

${ }^{a}$ Values of mass density, distance, mass and velocity are given in units of $M_{\odot} \mathrm{pc}^{-3}, \mathrm{kpc}, 10^{10} M_{\odot}$ and $\mathrm{km} \mathrm{s}^{-1}$, respectively. ${ }^{b}$ The corresponding value as in the WeCapp model is used. ${ }^{c}$ In the last row we give some relevant parameter values for the models MEGA A in de Jong et al. (2006).

mass function choice. For the lens mass in the M31 and MW halos, we assume the $\delta$-function approximation

$\psi_{0}(\mu)=\frac{\delta\left(\mu-\mu_{\mathrm{h}}\right)}{\mu_{\mathrm{h}}}$

and take a MACHO mass, in solar units, $\mu_{\mathrm{h}}=10^{-1}, 0.5,1$.

\subsection{Mass distributions in M 31 and MW}

The visible mass distributions for the M 31 bulge and disk are derived by fitting the observed brightness profiles given by Kent (1989) and by further assuming mass-to-light ratios for bulge and disk stellar populations. Moreover, the consideration of the M 31 rotation curve data allows us to derive the distribution of the dark matter in the M 31 halo.

Here, we use a coordinate system $(x, y, z)$ centred in M 31 , with $x$ axis along the disk major axis. We also assume that the disk is inclined by the angle $i=77^{\circ}$ and that the disk azimuthal angle relative to the near minor axis is $\phi=38.6^{\circ}$. The position angle of the bulge is $50^{\circ}$. We neglect the MW disk since we have verified that the expected number of events due to lenses belonging to this mass component is only about $1 \%$ of the total number of M 31 self-lensing events.

\subsubsection{31 bulge}

The M 31 bulge model is derived from Table 1 in Kent (1989) containing the bulge 3D brightness density in the Gunn $r$-band and the ellipticity $\epsilon(a)$ as a function of the major-axis distance $a$ to the M 31 centre.

We fit the 3D brightness profile with a single de Vaucouleurs $a^{1 / 4}$ law (reference model)

$j_{r}(a)=j_{r}(0) 10^{-0.4\left(7.598 a^{1 / 4}\right)} \quad a>a_{\min }$,

with central 3D brightness density $j_{r}(0)=9.57 \times$ $10^{-7} L_{\odot} \operatorname{arcsec}^{-3}$ (shifting to magnitudes, Eq. (19) may be written in the form $\left.m_{r}(a)=15.048+7.598 a^{1 / 4} \mathrm{mag} \operatorname{arcsec}^{-3}\right)$. This model accurately fits Kent data for $a_{\min } \simeq 1$ arcmin, namely in the region usually explored by pixel lensing observations.

From the 3D brightness density profile in Eq. (19), one can derive the corresponding mass density profile, which has the same behaviour as the brightness profile and central mass density given by

$\rho(0)=\left(\frac{M}{L_{R}}\right) 10^{-0.4\left[15.048-(r-R)-\mathrm{ext}_{R}-M_{\odot R}-d_{\bmod }\right]}$,

where $\left(M / L_{R}\right)$ is the mass-to-light ratio in the $R$-band, $(r-R)$ the colour of the bulge stellar population, $M_{\odot R}=4.42$ the absolute brightness of the Sun in the $R$-band, ext $_{R}$ the extinction in the same filter, and distance modulus $d_{\text {mod }}=24.43$ (for an M 31 distance of $770 \mathrm{kpc})$. By using the values $\left(M / L_{R}\right)=2.96,(r-$ $R)=0.59$ and $\operatorname{ext}_{R}=0.36$ quoted by Riffeser et al. (2006), we obtain $\rho(0)=4.53 \times 10^{4} M_{\odot} / \mathrm{pc}^{3}$, corresponding to a total bulge

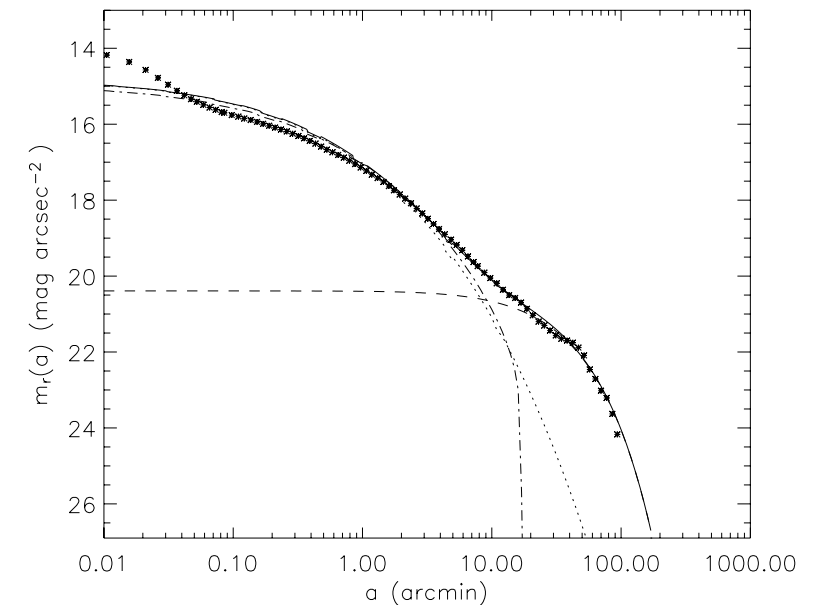

Fig. 1. The projected 2D brightness profile (solid line) for the reference model in comparison with Kent data (crosses).

mass $M_{\text {bulge }} \simeq 3.85 \times 10^{10} M_{\odot}$, in agreement with the value given by Kent (1989).

Note that the observed 2D brightness profile is also compatible with more concentrated mass distributions for the bulge (Beaton et al. 2006). For instance, we have tried a (boxy) model with $99 \%$ of the total mass inside $17.86 \operatorname{arcmin}(4 \mathrm{kpc})$. The mass density is now given by

$$
\begin{aligned}
\rho(a) & =4.40 \times 10^{4} 10^{-0.4\left(7.598 a^{0.24}\right)} \quad a \leq 17.86^{\prime} \\
& =1.81 \times 10^{39} 10^{-0.4\left(7.598 a^{0.90}\right)} \quad a>17.86^{\prime}
\end{aligned}
$$

In Fig. 1 the projected 2D brightness profile (in units of mag $\operatorname{arcsec}^{-2}$ ) is shown for reference model (solid line) together with Kent data (crosses). The dotted line gives the reference bulge and the dotted dashed line shows the bulge contribution of the boxy model. In deriving these profiles, we assumed that the bulge isophote are triaxial ellipsoids with semi-major axes

$a^{2}(\epsilon)=x^{2}+y^{2}+\frac{z^{2}}{(1-\epsilon)^{2}}$

and ellipticity varying on the semi-major axis according to the Kent data ${ }^{3}$.

From Fig. 1, one can see that beyond 0.03 arcmin both reference and boxy models accurately reproduce Kent data. The only difference is the behaviour of the bulge contribution at a large distance where, in any case, the disk contribution dominates.

3 The existing relation between $\epsilon$ and $a$ may be approximated by (Riffeser et al. 2006)

$\left(\frac{1}{1-\epsilon(a)}\right)^{2}=0.254 \frac{a}{\operatorname{arcmin}}+1.11$. 


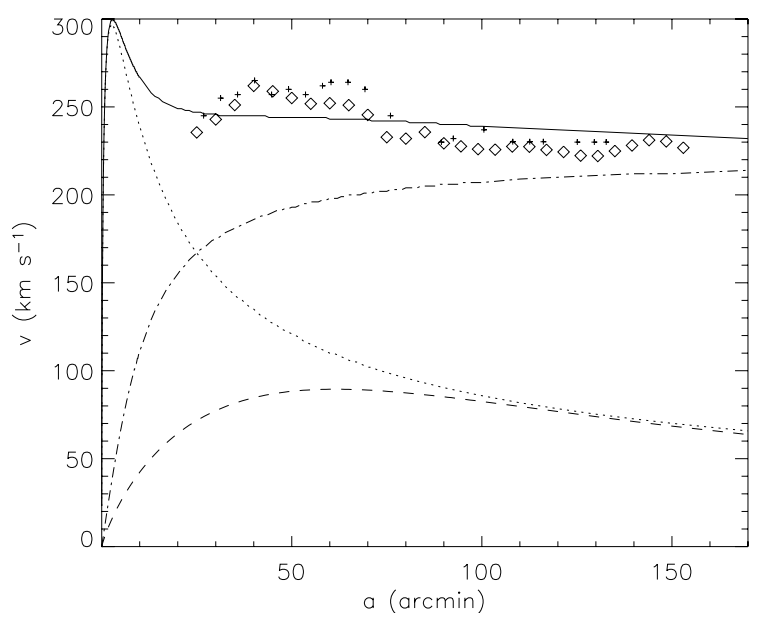

Fig. 2. The full M 31 rotation curve (solid line) in comparison with data points derived from Brinks \& Burton (1984) (crosses) and Carignan et al. (2006) (diamonds).

For comparison we also discuss the results obtained by using the bulge model adopted by the WeCapp collaboration (Riffeser et al. 2006).

\subsubsection{31 disk}

As in Kerins et al. (2001), the disk 3D brightness density in the $r$-band is modeled by the law

$j_{r}(x, y, z)=j_{r}(0) \exp \left(-\sqrt{x^{2}+y^{2}} / h\right) \operatorname{sech}^{2}(z / H)$,

and a best-fit procedure to the Kent data (for $a \gtrsim 6$ arcmin) allows to obtain the central brightness density $j_{r}(0)=4.2 \times$ $10^{-13} L_{\odot} \operatorname{arcsec}^{-3}$ (corresponding to a central magnitude $m_{r}(0)=$ 20.5), the radial scale length $h=27.95$ arcmin and the vertical scale length $H=1.34$ arcmin (corresponding to $h=6.4 \mathrm{kpc}$ and $H=0.3 \mathrm{kpc}$, respectively).

As for the bulge, the corresponding disk mass density profile follows the same behaviour as the brightness profile. Accordingly, the disk central mass density is derived by assuming the following parameter values $\left(M / L_{R}\right)=0.88,(r-R)=0.54$ and $\operatorname{ext}_{R}=0.68$ for the disk (Riffeser et al. 2006), implying $\rho(0)=0.2 M_{\odot} \mathrm{pc}^{-3}$ and a total disk mass $M \simeq 3.09 \times$ $10^{10} M_{\odot}$. The 2D disk brightness profile is also shown in Fig. 1 (dashed line).

\subsubsection{31 and MW halos}

Both M31 and MW halo mass distributions are modeled as isothermal spheres

$\rho(r)=\frac{\rho_{0}}{1+\left(\frac{r}{r_{0}}\right)^{2}}$.

For M31 a fit to the M31 rotational curve by using the threecomponent model (bulge, disk, and halo) allows us to get the best-fit parameter values $r_{0}=2 \mathrm{kpc}$ and $\rho(0)=0.23 M_{\odot} \mathrm{pc}^{-3}$ (see also Kerins et al. 2001 and Riffeser et al. 2006).

The overall M31 rotational curve and the contributions of the three components are shown in Fig. 2 together with data points derived from HI measurements of Brinks \& Burton (1984) (crosses) and Carignan et al. (2006) (diamonds). Dotted,

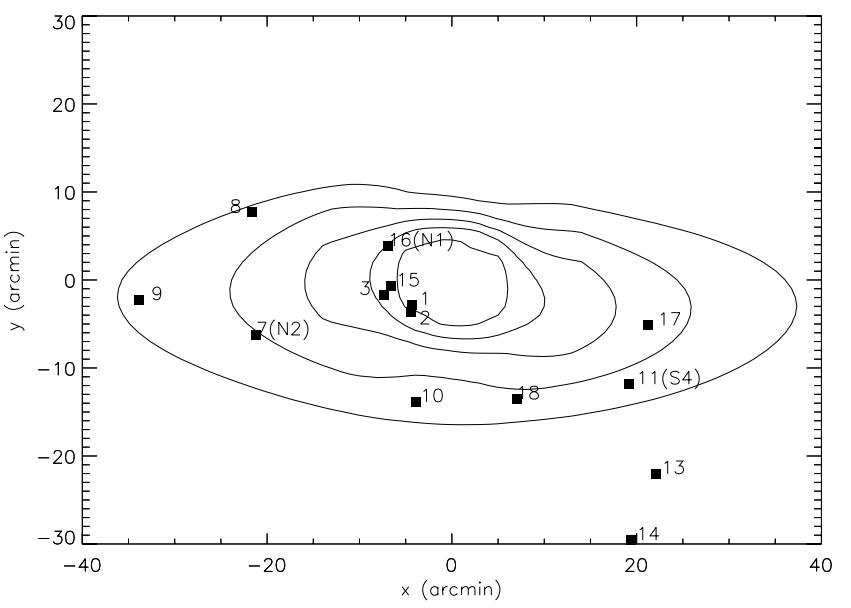

Fig. 3. The map $\mathrm{d} N_{\mathrm{ev}} / \mathrm{d} \Omega$ of the expected (total) event rate towards M 31 is shown, assuming the reference model, a MACHO mass value $\mu_{\mathrm{h}}=$ 0.5 and a MACHO halo dark matter fraction $f_{\mathrm{h}}=0.2$ (see text for details).

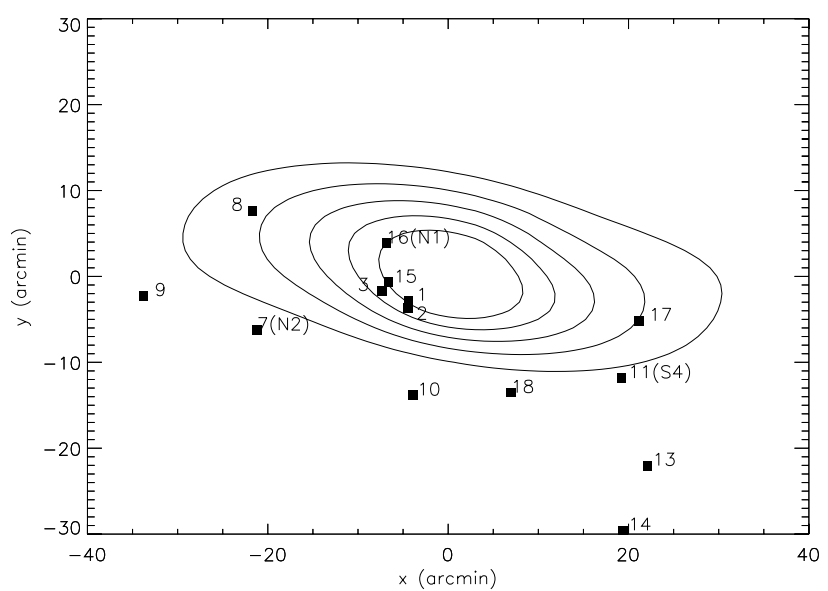

Fig. 4. The same as in Fig. 3 but for the dark-to-total event number ratio (see text details).

dashed and dot-dashed lines give the bulge, disk and halo contribution, respectively. In comparison with the recent determination of the mass distribution in M31 (Carignan et al. 2006), we find that at $R=35 \mathrm{kpc}$ the dark matter mass is $M_{\mathrm{h}}=$ $3.7 \times 10^{11} M_{\odot}$ and the stellar mass $M_{\mathrm{vis}}=6.6 \times 10^{10} M_{\odot}$. This translates to a total dynamical mass of $\simeq 4.4 \times 10^{11} M_{\odot}$ and to a rotational velocity of $233 \mathrm{~km} \mathrm{~s}^{-1}$ at $R=35 \mathrm{kpc}$, in agreement with the recent observations, and the M 31 halo is truncated at $R=150 \mathrm{kpc}$.

For the MW we use a core radius $a \simeq 5.6 \mathrm{kpc}$ and a local $\left(R_{0}=8.5 \mathrm{kpc}\right)$ dark matter density $\rho\left(R_{0}\right) \simeq 1.09 \times$ $10^{7} M_{\odot} \mathrm{kpc}^{-3}$. The corresponding asymptotic rotational velocity is $v_{\text {rot }} \simeq 220 \mathrm{~km} \mathrm{~s}^{-1}$. The MW halo is truncated at $R \simeq 100 \mathrm{kpc}$.

\subsection{Velocity dispersions}

The random velocities of stars and MACHOs are assumed to follow Maxwellian distributions, with one-dimensional velocity dispersion $\sigma=140$ and $166 \mathrm{~km} \mathrm{~s}^{-1}$ for the M 31 bulge and MACHOs, and $\sigma=156 \mathrm{~km} \mathrm{~s}^{-1}$ for the MACHOs in the MW halo. Moreover, following Widrow \& Dubinski (2005), the M 31 disk stars are assumed to have one-dimensional dispersion velocity decreasing towards the outer part from the central value $\sigma(r=0) \simeq 110 \mathrm{~km} \mathrm{~s}^{-1}$ to $\sigma(r=30 \mathrm{kpc}) \simeq 5 \mathrm{~km} \mathrm{~s}^{-1}$. In addition, 
Table 3. The MEGA event detection efficiency $\epsilon\left(t_{1 / 2}, \Delta f\right)$ is given as a function of $1 / \Delta f$ (first row) for different values of $t_{1 / 2}$ (first column) in days.

\begin{tabular}{ccccccccc}
\hline \hline & $0.02^{a}$ & 0.04 & 0.08 & 0.12 & 0.16 & 0.20 & 0.24 & 0.28 \\
\hline 1 & 0.09 & 0.08 & 0.02 & 0.01 & & & & \\
3 & 0.22 & 0.20 & 0.12 & 0.09 & 0.04 & 0.02 & & \\
5 & 0.24 & 0.21 & 0.14 & 0.08 & 0.04 & 0.02 & 0.01 & \\
10 & 0.29 & 0.30 & 0.30 & 0.19 & 0.12 & 0.06 & 0.02 & 0.01 \\
20 & 0.25 & 0.25 & 0.24 & 0.20 & 0.14 & 0.09 & 0.05 & 0.02 \\
50 & 0.14 & 0.16 & 0.22 & 0.21 & 0.19 & 0.15 & 0.12 & 0.08 \\
\hline
\end{tabular}

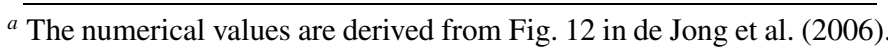

Table 4. The integrated number of expected events inside each iso-rate contour of Fig. 3 is given for self-lensing and dark-lensing.

\begin{tabular}{ccccc}
\hline \hline $\begin{array}{l}\text { Events inside } \\
\text { the } 8 \text { MEGA } \\
\text { fields }\end{array}$ & & & & \\
\hline & $\begin{array}{c}\text { iso-rate contour } \\
\text { event arcmin }\end{array}$ & self & dark & MEGA $^{a}$ \\
& $1 \times 10^{-1}$ & 3.80 & 0.90 & 1 \\
& $3 \times 10^{-2}$ & 6.49 & 2.61 & 4 \\
reference $^{b}$ & $2 \times 10^{-2}$ & 7.45 & 3.79 & 5 \\
& $1 \times 10^{-2}$ & 8.60 & 6.77 & 6 \\
& $5 \times 10^{-3}$ & 9.20 & 9.68 & 12 \\
\hline & overall & 9.68 & 11.76 & 14 \\
\hline
\end{tabular}

$a$ The quoted values refer to the number of events detected by the MEGA collaboration. ${ }^{b}$ Here, we are assuming the reference model with $\mu_{\mathrm{h}}=0.5$ and $f_{\mathrm{h}}=0.2$.

a rigid rotational velocity of $40 \mathrm{~km} \mathrm{~s}^{-1}$ has been taken into account for the M 31 bulge (Kerins et al. 2001; An et al. 2004). For the M 31 disk component, the full rotational velocity is also considered as shown in Fig. 2 (solid line).

\section{Results and concluding remarks}

The main purpose of the present analysis is to compare the predictions of our model with the observational results obtained by the MEGA collaboration (de Jong et al. 2006). Therefore, to evaluate the microlensing rate, we reproduce the MEGA observational setup and make use of the event detection efficiency $\epsilon\left(t_{1 / 2}, \Delta f\right)$, as a function of the time duration and amplification at maximum and of the maximum impact parameter $u_{\mathrm{T}}(M)$ values as given by de Jong et al. (2006). In Table 3 we give typical detection efficiency values derived from Fig. 12 in de Jong et al. (2006). In order to take the spatial variation of the detection efficiency into account we use two different evaluations of $\epsilon$ at distances both smaller and larger than 11 arcmin from the M 31 centre $^{4}$. This leads to $\epsilon$ respectively smaller and larger on average by about $30 \%$ of the values quoted in Table 3 .

In the following tables and figures, we assume for both the M 31 and MW halos a MACHO halo dark matter fraction $f_{\mathrm{h}}=0.2$, as suggested by microlensing observations towards the Magellanic Clouds (Alcock et al. 2000) and pixel-lensing observations towards M 31 (Calchi Novati et al. 2005). However, most of our results can be easily rescaled to other values of $f_{\mathrm{h}}$. In Table 6 we consider different values for the MACHO mass: $\mu_{\mathrm{h}}=0.1,0.5$, and 1 (in solar units). Figures 3-6 and Tables 4, 7 , and 8 are given for $\mu_{\mathrm{h}}=0.5$.

\footnotetext{
${ }^{4}$ De Jong, private communication.
}

Table 5. Number of self-lensing events expected given the setup of the MEGA campaign, for the different models discussed in the text. We consider different source and lens populations.

\begin{tabular}{cccccc}
\hline \hline $\begin{array}{l}\text { Events inside } \\
\text { the 8 MEGA } \\
\text { fields }\end{array}$ & & & & & \\
\hline & bb & bd & db & dd & self \\
reference & 4.25 & 1.17 & 3.30 & 0.96 & 9.68 \\
boxy & 5.14 & 1.10 & 2.76 & 0.95 & 9.95 \\
WeCapp & 4.98 & 1.34 & 4.08 & 0.96 & 11.37 \\
\hline
\end{tabular}

Table 6. For the reference model, the expected number of dark-lensing events is given for $\mu_{\mathrm{h}}=0.1,0.5,1$ and $f_{\mathrm{h}}=0.2$.

\begin{tabular}{ccccccc}
\hline \hline $\begin{array}{l}\text { Events inside } \\
\text { the } 8 \text { MEGA } \\
\text { fields }\end{array}$ & & & & & & \\
\hline & $\mu_{\mathrm{h}}$ & $\mathrm{bh}$ & $\mathrm{bH}$ & $\mathrm{dh}$ & $\mathrm{dH}$ & dark \\
\hline reference & 0.1 & 2.55 & 1.04 & 8.81 & 3.10 & 14.49 \\
& 0.5 & 1.96 & 0.72 & 6.85 & 2.23 & 11.76 \\
& 1 & 1.68 & 0.58 & 5.80 & 1.82 & 9.88 \\
\hline
\end{tabular}

Assuming the reference model for the M31 mass distribution, the spatial distribution of the expected events is shown in Figs. 3 and 4. Here we give maps in the sky plane of the (total) event rate and dark-to-total event number ratio, respectively. We also adopt the observational parameters of the MEGA collaboration. Accordingly, we consider $T_{\mathrm{obs}}=2 \mathrm{yr}$ and we account for the detection efficiency $\epsilon\left(t_{1 / 2}, \Delta f\right)$ and maximum impact parameter $u_{\mathrm{T}}(M)$ as given by de Jong et al. (2006). In particular, in Fig. 3, from the outer to the inner M 31 region, contour levels correspond to the values $5 \times 10^{-3}, 1 \times 10^{-2}, 2 \times 10^{-2}, 3 \times$ $10^{-2}, 1 \times 10^{-1}$ event $\operatorname{arcmin}^{-2}$, respectively. In Fig. 4, from the inner to outer region, contour levels correspond to the values $0.4,0.5,0.6,0.7$ and 0.8 , respectively.

In Table 4 we give our estimate of the integrated number of expected events inside each iso-rate contour of Fig. 3. Here and in the following we consider events inside the 8 fields selected by the MEGA collaboration (as reported in Fig. 15 in de Jong et al. (2006) the innermost M 31 region is excluded). From Fig. 3 and Table 4, one can see that dark-lensing makes an important contribution to pixel-lensing beyond the second (from the inner) iso-rate contour, namely beyond $\simeq 10$ arcmin from the M 31 centre.

The expected number of self-lensing events inside the 8 MEGA fields is given in Table 5 for different source and lens populations. Here with the symbols $\mathrm{b}, \mathrm{d}$, and $\mathrm{h}$ we indicate sources and/or lenses in the M31 bulge, disk, and halo, respectively. Capital symbol $\mathrm{H}$ is used to indicate lenses in the MW halo. In any case, the first (second) symbol refers to the source (lens). From Table 5 one can see that, for all the considered models (reference, boxy and WeCapp), the total number of selflensing events is roughly the same (within 15\%).

For the reference and boxy models, we note an increase in bulge-bulge events to compensate for a decrease in disk-bulge ones. This is expected to be due to the different concentrations of bulge mass for the two distributions. We also note the increase in the disk-bulge events in the WeCapp model due to the more extended bulge mass distribution.

Assuming the reference model and $f_{\mathrm{h}}=0.2$, in Table 6 we give our estimate of the expected number of dark-lensing events for several MACHO mass values. We find that the total number of dark-lensing and self-lensing events turns out to be roughly 
Table 7. The number of self-lensing and dark M31-lensing (due to MACHOs in the M31 halo) events for our two models (here labeled reference A and boxy A) assuming $M_{\mathrm{b}}=4.4$ and $M_{\mathrm{d}}=5.5$ (in units of $\left.10^{10} M_{\odot}\right)$ and $\mu_{\mathrm{h}}=0.5, f_{\mathrm{h}}=0.2$.

\begin{tabular}{ccccc}
\hline \hline $\begin{array}{l}\text { Events inside } \\
\text { the 8 MEGA } \\
\text { fields }\end{array}$ & self & $\begin{array}{c}\text { dark } \\
\text { M31 }\end{array}$ & self & $\begin{array}{c}\text { dark } \\
\text { M31 }\end{array}$ \\
\hline & $\begin{array}{c}H=0.3 \\
(\mathrm{kpc})\end{array}$ & $\begin{array}{c}H=0.3 \\
(\mathrm{kpc})\end{array}$ & $\begin{array}{c}H=1 \\
(\mathrm{kpc})\end{array}$ & $\begin{array}{c}H=1 \\
(\mathrm{kpc})\end{array}$ \\
\hline reference A & 12.4 & 8.8 & 15.5 & 8.6 \\
boxy A & 12.7 & 8.5 & 15.5 & 8.7 \\
MEGA A2 & - & - & 12.4 & 5.7 \\
MEGA A1 & - & - & 14.2 & 6.2 \\
\hline
\end{tabular}

the same. The total (self + dark+background) number of expected events is $\sim 23$ including $\sim 1$ event due to supernovae (SN) contamination. This is consistent at a $2 \sigma$ confidence level with the 14 candidate MEGA events assumed to follow a Poisson distribution.

A comparison of our results with the corresponding values reported in Table 5 of de Jong et al. (2006) for low and high internal extinction ${ }^{5}$ shows that there is fairly good agreement. Indeed, to get a more meaningful comparison for the self-lensing contribution, we normalised the values for the mass of the luminous components to those of the MEGA models (e.g. for their models A, $M_{\mathrm{b}}=4.4 \times 10^{10} M_{\odot}$ and $\left.M_{\mathrm{d}}=5.5 \times 10^{10} M_{\odot}\right)$ and used a more broadened disk $(H=1 \mathrm{kpc})$. In Table 7 we report the obtained results for our models (reference and boxy, now labeled A) with the same bulge and disk mass as in MEGA models A, for two values of the disk-scale height $H=0.3 \mathrm{kpc}$ and $H=1 \mathrm{kpc}$. We refer to models with $M_{\mathrm{d}}=5.5 \times 10^{10} M_{\odot}$ and $H=1 \mathrm{kpc}$ as maximal disk models. In the last row we report some results from Table 5 in de Jong et al. (2006), for the MEGA models in the case of high (MEGA A2) and low (MEGA A1) extinction and for a 20\% M 31 MACHO halo. Hence, we can see that our estimate of the (total) number of the self-lensing events agrees with the de Jong et al. (2006) prediction only when considering more extreme (maximal) parameters for the disk component ${ }^{6}$.

At variance with de Jong et al. (2006) we nevertheless do not conclude that all the 14 events detected by the MEGA collaboration can be explained only by self-lensing. Indeed, the spatial distribution of the events occurring inside the 8 MEGA fields ${ }^{7}$ clearly shows that the distribution with the distance from the M31 centre of the self-lensing events hardly can be reconciled with the MEGA data.

In Fig. 5, for the reference (dotted line) and boxy (dashed line) models, the (normalized) distribution of the expected number of self-lensing events within the 8 MEGA fields is given as a function of the the distance from the M31 centre. The same

\footnotetext{
${ }^{5}$ Note that we are considering a total extinction in the $r$-band of $0.36 \mathrm{mag}(0.68 \mathrm{mag})$ for the bulge (disk), irrespective of the line of sight.

${ }_{6}$ As concerns our estimate in Table 7 of dark-lensing events due to the M 31 halo, we obtained a larger number of events with respect to MEGA expectations $\left(\simeq 9\right.$ events instead of $\simeq 6$ events for $m_{\mathrm{h}}=0.5$ and $f_{\mathrm{h}}=0.2$ ). However, to describe the M 31 dark matter halo we are adopting a different density law (an isothermal profile truncated at $R=150 \mathrm{kpc}$ ), which is in any case consistent with the full M 31 rotation curve.

7 The spatial distribution of the events observed within the 8 MEGA fields is given in Table 8 for several models (reference, boxy and WeCapp) and shown (normalized to unity) in Fig. 5 for both self-lensing (reference and boxy) and total (self+dark) lensing (reference).
}

Table 8. Distribution of the number of self-lensing events with the distance from the M31 centre for several models.

\begin{tabular}{ccccc}
\hline \hline $\begin{array}{c}\text { Events inside } \\
\text { the } 8 \text { MEGA } \\
\text { fields }\end{array}$ & Ref. & Box. & Wec. & Ref. $^{a}$ \\
\hline$d($ arcmin $)$ & self & self & self & dark \\
\hline $2-5$ & 3.81 & 4.55 & 3.92 & 0.93 \\
$5-10$ & 2.80 & 3.22 & 3.43 & 1.97 \\
$10-15$ & 1.32 & 1.06 & 1.73 & 2.46 \\
$15-20$ & 0.66 & 0.27 & 0.88 & 2.20 \\
$20-25$ & 0.42 & 0.17 & 0.55 & 1.78 \\
$25-30$ & 0.22 & 0.11 & 0.28 & 1.23 \\
$30-35$ & 0.09 & 0.06 & 0.12 & 0.78 \\
$35-40$ & 0.04 & 0.03 & 0.05 & 0.33 \\
\hline
\end{tabular}

${ }^{a}$ In the last column, the same quantity is given for dark-lensing assuming the reference model, $\mu=0.5$ and $f_{\mathrm{h}}=0.2$.

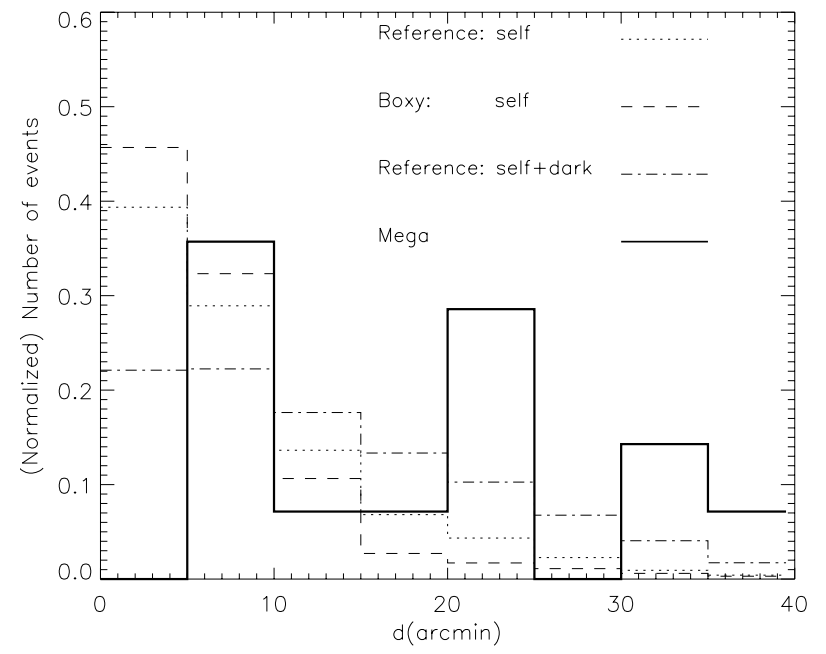

Fig. 5. The normalised distribution of the expected number of selflensing events within the 8 MEGA fields as a function of the the distance from the M 31 centre.

quantity is shown for self+dark lensing (thin solid line) assuming the reference model, $f_{\mathrm{h}}=0.2$ and $\mu_{\mathrm{h}}=0.5$. For comparison the (normalized) distribution of the 14 observed MEGA events is also given (thick solid line). An excess of events with respect to expectations from self-lensing remains at a large distance. This conclusion is enhanced when assuming the boxy model for the M31 bulge.

A better agreement with MEGA data can be obtained if one also considers a dark-lensing (with $\mu_{\mathrm{h}}=0.5$ and $f_{\mathrm{h}}=0.2$ ) contribution. The compatibility between the observed MEGA event distribution as a function of distance from the M 31 centre and the expected one has been evaluated for both self-lensing and the self+dark lensing hypotheses ${ }^{8}$. By using the KolmogorovSmirnov test (Press et al. 1986), we find a K-S probability $\simeq 0.51$ for self+dark lensing and $\simeq 0.18$ for self-lensing only, thus implying that a dark matter contribution to microlensing seems to be favoured.

However, we caution that the candidate microlensing events could be contamined by variable stars. In particular, the events labelled 13 and 14, located in a region where the microlensing rate is negligible, might be contaminated by background

8 The comparison has been done excluding one event from the MEGA candidate list (in the exterior region) since we expect that at least one of them is due to the contamination of background supernovae (see text for more details). 


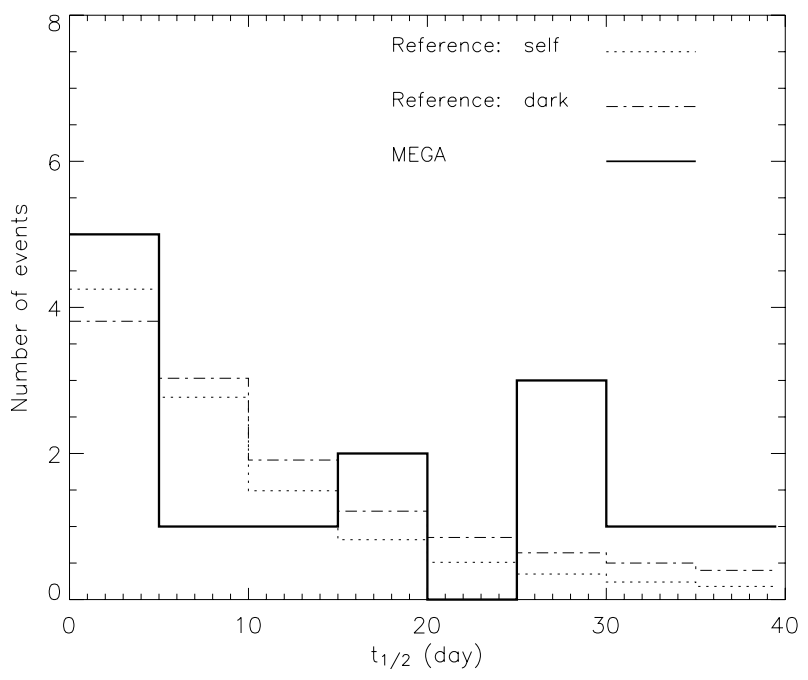

Fig. 6. The expected event number within the 8 MEGA fields as a function of $t_{1 / 2}$, for both self-lensing (dotted line) and dark-lensing (dotdashed line) in the case of the reference model. For comparison the distribution with $t_{1 / 2}$ of the 14 observed MEGA events is also given.

supernovae. Indeed, by assuming standard SN rate (Cox 2000) and integrating over the volume within $z_{\max } \simeq 0.4$ (the maximum distance at which the SN signal-to-noise ratio is at least $3 \sigma$ above the typical baseline of $22 \mathrm{mag} \mathrm{arcsec}^{-2}$ ), we expect about one detectable $\mathrm{SN}$ in the outer M 31 regions during the observational MEGA campaign.

The distribution of the expected number of events with the time scale $t_{1 / 2}$ is shown in Fig. 6 for the reference model and $f_{\mathrm{h}}=0.2$, and $\mu_{\mathrm{h}}=0.5$. From this figure, one can see that selflensing and dark-lensing events almost have the same $t_{1 / 2}$ distribution. Therefore, the $t_{1 / 2}$ event distribution is not particularly useful for determining the nature of the 14 MEGA events, at least for a MACHO mass value near $0.5 M_{\odot}$ (see also Ingrosso et al. 2006). The excess of long duration events in the MEGA data also suggests a contamination by other variable objects.

We emphasise that our analysis shows that hardly any 14 MEGA events can be due to self-lensing events by M 31 stars. On the other hand, given the few events detected up to now, it also seems premature to estimate the halo dark matter fraction in form of MACHOs.

Acknowledgements. We thank the referee for useful comments. G.I., F.D.P., and A.A.N. were partially supported by MIUR through PRIN 2004 prot. 2004020323_004. S.C.N. and G.S. were partially supported by MIUR through PRIN 2004 - prot. 2004024710_006. S.C.N. and Ph.J. thank the Swiss National Science Foundation for support.

\section{References}

Afonso, C., Albert, J. N., Andersen, J., et al. 2003, A\&A, 400, 951 Alcock, C., Akerloff, C. W., Allsman, R. A., et al. 1993, Nature, 365, 621 Alcock, C., Allsman, R. A., Alves, D. R., et al. 2000, ApJ, 542, 281 An, J. H., Evans, N. W., Kerins, E., et al. 2004, ApJ, 601, 845 Ansari, R., Auriere, M., Baillon, P., et al. 1997, A\&A, 324, 843 Ansari, R., Auriere, M., Baillon, P., et al. 1999, A\&A, 344, L49 Aubourg, E., Bareyre, P., Brehin, S., et al. 1993, Nature, 365, 623 Auriere, M., Baillon, P., Bouquet, A., et al. 2001, ApJ, 553, L137 Baillon, P., Bouquet, A., Giraud-Heraud, Y., \& Kaplan, J. 1993, A\&A, 277, 1 Baltz, E. A., Gyuk, G., \& Crotts, A. P. S. 2003, ApJ, 582, 30 Beaton, R. L., Majewski, S. R., Guhathakurta, P., et al. 2006 [arXiv: astro-ph/0605239]

Belokurov, V., An. J., Evans, N. W., et al. 2005, MNRAS, 357, 17 Bender, R., Kormendy, J., Bower, G., et al. 2005, ApJ, 631, 280 Brinks, E., \& Burton, W. B. 1984, A\&A, 141, 195 Binney, J., \& Tremaine, S. 1987, Galactic Dynamics (Princeton, New Jersey: Princeton University Press)

Calchi Novati, S., Iovane, G., Marino, A., et al. 2002, A\&A, 381, 848 Calchi Novati, S., Jetzer, Ph., Scarpetta, G., et al. 2003, A\&A, 405, 851 Calchi Novati, S., Paulin-Henriksson, S., Baillon, P., et al. 2005, A\&A, 443, 911 Carignan, C., Chemin, L., Huchtmeier, W. K., \& Lockman, F. J. 2006, AJ, 641, L109

Cox, A. N., ed. 2000, Allen's Astrophysical Quantities (Springer) Crotts, A. P. S. 1992, ApJ, 399, L43

Crotts, A. P. S., \& Tomaney, A. B. 1996, ApJ, 473, L87

de Jong, J. T. A., Kuijken, K., Crotts, A. P. S., et al. 2004, A\&A, 417, 461 de Jong, J. T. A., Widrow, L. M., Cseresnjes, P., et al. 2006, A\&A, 446, 855 De Paolis, F., Ingrosso, G., Nucita, A., \& Zakharov, A. F. 2005, A\&A, 432, 501 De Rújula, A., Jetzer, Ph., \& Masso, E. 1991, MNRAS, 250, 348 Gould, A. 1996, ApJ, 470, 201

Gould, A., Bahcall, J. N., \& Flynn, C. 1997, ApJ, 482, 913

Griest, K. 1991, ApJ, 366, 412

Ingrosso, G., Calchi-Novati, S., De Paolis, F., et al. 2006, A\&A, 445, 375 Jetzer, Ph. 1994, A\&A, 286, 426

Jetzer, Ph., Mancini, L., \& Scarpetta, G. 2002, A\&A, 393, 129

Joshi, Y. C., Pandey, A. K., Narasimha, D., \& Sagar, R. 2005, A\&A, 433, 787

Kent, S. M. 1989, AJ, 97, 1614

Kerins, E. 2004, MNRAS, 347, 1033

Kerins, E., Carr, B., Ewans, N. W., et al. 2001, MNRAS, 323, 13

Kerins, E., An, J., Ewans, N. W., et al. 2003, ApJ, 598, 993

Kerins, E., Darnley, M. J., Duke, J., et al. 2006, MNRAS, 365, 1099

Mamon, G. A., \& Soneira, R. M. 1982, ApJ, 255, 181

Paczyński, B. 1986, ApJ, 304, 1

Paulin-Henriksson, S., Baillon, P., Bouquet, A., et al. 2003, A\&A, 405, 15

Press, W. H., Teukolsky, S. A., Vetterling, W. T., \& Flannery, B. P. 1986,

Numerical recipes (Cambridge University Press)

Riffeser, A., Fliri, J., Bender, R., et al. 2003, ApJ, 599, L17

Riffeser, A., Fliri, J., Seitz, S., \& Bender, R. 2005, ApJS, 163, 225

Tisserand, P., \& Milsztajn, A. 2005 [arXiv: astro-ph/0501584]

Tisserand, P., Le Guillou, L., Afonso, C., et al. 2006

[arXiv: astro-ph/0607207]

Udalski, A., Szymanski, M., Kaluzny, J., et al. 1993, Acta Astron., 43, 289

Widrow, L. M., \& Dubinski, J. 2005, ApJ, 631, 838

Zoccali, M., Cassisi, S., Frogel, J. A., et al. 2000, ApJ, 530, 418 